\title{
AS VANTAGENS DO BENCHMARKING AMBIENTAL
}

Autor: Marilena Lino de Almeida Lavorato, Publicitária (PUCC- Pontíficia Universidade Católica de Campinas), pós-graduada em Marketing (ESPM- Escola Superior de Propaganda e Marketing ), Sociologia e Política (EPGSP- Escola Pós Graduada de Sociologia e Política de São Paulo ), Gestão Estratégica de Negócios (FGV - Fundação Getúlio Vargas) e Gestão Ambiental (IETEC - Instituto de Educação Tecnológica). Diretora Executiva da MAIS Projetos (gestão sócioambiental) e Coordenadora do GMGA - Grupo Multidisciplinar Gestão Ambiental (Apoio APARH- Associação Paulista de Administração de Recursos Humanos) .

Email: marilena@maisprojetos.com.br Site: www.maisprojetos.com.br

Resumo: Este artigo discorre sobre a adoção do Benchmarking Ambiental para o aprimoramento técnico-gerencial da gestão ambiental corporativa como ferramenta de melhoria contínua e aumento de competitividade para as organizações. Aborda a percepção empresarial em relação ao tema, reconhecendo a gestão ambiental como um dos pilares do crescimento sócio-econômico. E dentro deste contexto aponta o Benchmarking Ambiental Brasileiro como opção de crescimento e amadurecimento de nosso modêlo empresarial, opção esta confirmada pelas pesquisas do Prof. D. Enrique de la Rica, Director de la Escuela Europea de Estudios Universitarios y de Negocios (ESEUNE www.eseune.edu Neguri, Getxo - Bizkaia - España), que afirma "processos de melhoria contínua sem a pratica do Benchmarking conseguem em torno de 7\% de melhoria em suas ações. Já com a inclusão da prática do Benchmarking, conseguem encontrar fórmulas que representam até 300\% de melhoria".

Palavras Chaves: Benchmarking Ambiental, Gestão Ambiental, Desenvolvimento Sustentável, Educação Ambiental Corporativa 


\title{
THE ADVANTAGES OF THE BENCHMARKING ENVIRONMENTAL
}

\author{
Author: Marilena Lino de Almeida Lavorato, Advertising (PUCC- Pontíficia \\ Catholic University), postgraduate in Marketing (ESPM- Publicity Superior School \\ and Marketing), Sociology and Politics (EPGSP-School Powders Graduated of \\ Sociology and Politics of S. Paulo), Strategic Management of Business (FGV - \\ Foundation Getúlio Vargas) and Environmental Management (IETEC - \\ Technological Institute of Education). Director Executive from the \\ MAISPROJETOS (partner-environmental management) and Coordinator of the \\ GMGA - Group Several Disciplinary Environmental Management (Support \\ APARH- Human Resorts Administration Association). Email: \\ marilena@maisprojetos.com.br Site: www.maisprojetos.com.br
}

Summary: This article discourse about the adoption of the Benchmarking Environmental for improve the technical-managerial from the corporate environmental management as continuous tool of improvement and I increase of competitiveness for the organizations. It approaches the business perception regarding fear, recognizing the environmental management as one of the pikes of the socio-economic growth. And inside this context aims the Benchmarking Environmental Brazilian as option of growth and maturation of our format business, option this confirmed by the researches of the Prof. D. Enrique de La Rica, Director of the Europe School Studies (ESEUNE www.eseune.edu - Neguri, Getxo - Bizkaia - Spain), that affirms "continuous trials of improvement without the practices of the Benchmarking they obtain around 7\% of improvement in. Already with the enclosure from the practice of the Benchmarking, they obtain find formulae that represent to $300 \%$ of improvement".

Words Keys: Benchmarking Environmental, Environmental Management, Sustainable Development, Corporate Environmental Education 


\section{INTRODUÇÃO:}

A partir da escassez dos recursos naturais, somado ao crescimento desordenado da população mundial e intensidade dos impactos ambientais, surge o conflito da sustentabilidade dos sistemas econômico e natural, e faz do meio ambiente um tema literalmente estratégico e urgente para as empresas e organizações. O homem começa a entender a impossibilidade de transformar as regras da natureza e a importância da reformulação de suas práticas ambientais.

Durante o período da chamada Revolução industrial não havia preocupação com a questão ambiental. Os recursos naturais eram abundantes, e a poluição não era foco da atenção da sociedade industrial e intelectual da época.

A partir da escassez dos recursos naturais, somado ao crescimento desordenado da população mundial e intensidade dos impactos ambientais, surge o conflito da sustentabilidade dos sistemas econômico e natural, e faz do meio ambiente um tema literalmente estratégico e urgente. O homem começa a entender a impossibilidade de transformar as regras da natureza e a importância da reformulação de suas práticas ambientais.

\subsection{Os limites}

A começar pelos limites físicos. Estamos consumindo $20 \%$ a mais de recursos naturais do que o planeta é capaz de repor, e assim, estamos avançando sobre os estoques naturais da Terra, comprometendo as gerações atual e futuras segundo o Relatório Planeta Vivo 2002, elaborado pelo WWF e lançado ano passado em Genebra.

De acordo com este o relatório, o planeta tem 11,4 bilhões de hectares de terra e espaço marinho produtivos - ou 1,9 hectares de área produtiva per capita. Mas a humanidade está usando o equivalente a 13,7 bilhões de hectares para produzir os grãos, peixes e crustáceos, carne e derivados, água e energia que consome. Cada um dos 6 bilhões de habitantes da Terra, portanto, usa uma área de 2,3 hectares. Essa 
área é a Pegada Ecológica de cada um. O fator de maior peso na composição da Pegada Ecológica hoje é a energia, sobretudo nos países mais desenvolvidos.

A Pegada Ecológica de 2,3 hectares é uma média. Mas há grandes diferenças entre as nações mais e menos desenvolvidas, como mostra o Relatório Planeta Vivo, que calculou a Pegada de 146 países com população acima de um milhão de habitantes. Os dados mais recentes (de 1999) mostram que enquanto a Pegada média do consumidor da África e da Ásia não chega 1,4 hectares por pessoa, a do consumidor da Europa Ocidental é de cerca de 5,0 hectares e a dos norte-americanos de 9,6 hectares.

Embora a Pegada brasileira seja de 2,3 hectares - dentro da média mundial, mas cerca de $20 \%$ acima da capacidade biológica produtiva do planeta.

Quando falamos em emissões de poluentes, as diferenças dos índices emitidos pelos países desenvolvidos e em desenvolvimento também são significativas: Um cidadão médio norte-americano, por exemplo, responde pela emissão anual de 20 toneladas anuais de dióxido de carbono; um britânico, por 9,2 toneladas; um chinês, por 2,5; um brasileiro, por 1,8; já um ganês ou um nicaragüense, só por 0,2 ; e um tanzaniano, por 0,1 tonelada anual. A China e o Leste da Ásia aumentaram em $100 \%$ o consumo de combustíveis fósseis em apenas cinco anos (1990/95). (Wolfgang Sachs, do Wuppertal Institute)

Nos países industrializados cresce cada vez mais o consumo de recursos naturais provindos dos países em desenvolvimento - a ponto de aqueles países já responderem por mais de $80 \%$ do consumo total no mundo. Segundo Sachs, 30\% dos recursos naturais consumidos na Alemanha vêm de outros países; no Japão, 50\%; nos países Baixos, $70 \%$.

Somado ao escasseamento dos recursos naturais pelo consumo, temos também a sua degradação intensificada pelos acidentes ecológicos que acelera a urgência da questão ambiental.

\section{A EVOLUÇÃO DA PERCEPÇÃO AMBIENTAL NA EMPRESA:}


Dentro desta nova configuração, a empresa passou a viver o conflito da sustentabilidade dos sistemas econômico e natural, que visto sob esta ótica, são excludentes, e faz do meio ambiente um tema literalmente estratégico dentro das organizações.

O maior desafio das organizações que é manter e aumentar a competitividade e ao mesmo tempo atender as pressões dos stakeholders (públicos de interesse), ficou bem mais complexo com a inclusão da variável ambiental. Neste novo cenário, surgiram outras formas de pressões e outras categorias de stakeholders.

Uma nova postura se fez necessária, e uma relação mais estreita foi estabelecida imposta pelos limites dos sistemas (natural e econômico), começando aí o entendimento da importância do meio ambiente nas questões empresariais. Como resultado desta preocupação, tivemos na empresa, o surgimento dos SGAs - Sistemas de Gestão Ambiental e a adoção do Benchmarking Ambiental nas empresas.

\section{3- BENCHMARKING:}

É um processo sistemático e contínuo para avaliar produtos, serviços e processos de trabalho das organizações que são reconhecidas como representantes das melhores práticas com o propósito de realizar melhorias organizacionais.

Benchmarking é também uma ferramenta de gestão para propor metas, utilizando-se de normas externas e objetivas para aprender novos processos de gestão. Segundo o autor Roberto J. Boxwell em seu livro "Benchmarking, competir com vantagens", Benchmarking trata-se de uma ferramenta que serve descobrir, analisar, comparar, aprender com empresas líderes em seus segmentos, métodos e processos competitivos, e assim adaptar este novo conhecimento nos próprios processos para aumentar a competitividade de seu negócio. Benchmarking é hoje reconhecido no meio empresarial como um método essencial para a prática da melhoria contínua.

Empresas inteligentes estão passando para o benchmarking de ciclo rápido. "Ele é hoje um processo mais rápido e flexível para as empresas que desejam aumentar seus conhecimentos e tornar a administração bem-sucedida".diz o professor David 
Garvin, responsável pelo programa de Estratégias de Gestão da Harvard Business Scholl. Para Garvin, o novo benchmarking é mais prático pois permite as empresas identificarem problemas operacionais específicos e novas oportunidades.

Em outras palavras, Benchmarking é o processo de identificar, compreender e adaptar os processos e práticas de outras organizações para ajudar na melhoria de nossa empresa. Benchmarking não é espionagem, cópia, etc. É aprendizado. É uma postura humilde e racional, pois é uma forma de admitir que outra organização é melhor em algum processo e que por meio da observação e comparação podemos aprender com seus êxitos.

Enfim, Benchmarking é considerado um saudável exercício empresarial na medida em que troca, soma, reconhece, gera vínculos, respeita a multidiversidade, integra setores, constrói novos conhecimentos, e principalmente, cria o hábito das relações éticas, transparentes e solidárias entre empresas e seus diversos segmentos.

\subsection{Vantagens do Benchmarking:}

Por que reinventar soluções competitivas já existentes investindo tempo e dinheiro. É mais racional, partir do já existente para desenvolvimento e/ou adaptações. Segundo o Prof. D. Enrique de la Rica, Director de la Escuela Europea de Estudios Universitarios y de Negocios (ESEUNE www.eseune.edu Neguri, Getxo - Bizkaia - España), processos de melhoria contínua sem a pratica do Benchmarking conseguem em torno de 7\% de melhoria em seus ações. Já com a inclusão da prática do Benchmarking, conseguem encontrar fórmulas que representam até $300 \%$ de melhoria.

\subsection{Tipos de Benchmarking}

Podemos classificar Benchmarking em 2 formas de atuação: interno e externo.

O Benchmarking Interno se baseia em análise das organizações, produtos e serviços dentro de nossa própria empresa. Exemplos: Marketing pode aprender técnicas de gestão com a Produção e vice-versa. Uma comparação de processos entre áreas. Num mercado globalizado, pode-se e deve-se aprender com os sucessos das diversas 
unidades. Por exemplo: A unidade de determinado país ensinando processos competitivos para outras unidades de outros países. Lembrem-se Benchmarking trata-se de aprender com os mais competitivos em determinado processo.

O Benchmarking externo pode se dividir em 2 tipos: Competitivo e Global

3.2.1. Benchmarking Competitivo se baseia na análise e comparação da competência. Isto se faz com a análise da performance de outras instituições. É comum, monitorarmos a concorrência e aprendermos com ela. O mesmo se dá em relação as organizações que apresentam resultados significativos e transformam-se em cases de sucesso. Fica obrigatório o conhecimento, a análise e a comparação com elas. E isto é benchmarking.

3.2.2. Benchmarking Global é aquele que procura soluções fora do seu mercado. Procura os melhores mundialmente (líderes mundiais em seus campos de atuação) e assim faz seu processo de análise, comparação e adaptação, mesmo que virtualmente.

\section{3 - Etapas para a realização de Benchmarking:}

1. Definir a área, o propósito e os objetivos de Benchmarking

2. Criar uma equipe de trabalho

3. Identificar as instituições que vamos estudar

4. Definir uma metodologia de trabalho (coleta e organização de dados)

5. Realizar a coleta de dados

6. Fazer a comparação estabelecendo as principais diferenças entre nossos processos e os processos da organização estudada

7. Implementar um novo processo (adaptação e desenvolvimento a partir do aprendizado adquirido)

Através do Benchmarking podemos fazer a comparação de indicadores chaves tais como: identificar áreas competitivas em suas respectivas organizações, principais diferenças e fragilidades em relação a nossas organizações, estimar o impacto financeiro destas fragilidades e priorizar áreas para melhoria de resultados, etc. 
Normalmente, as principais áreas de interesse típico para um estudo de Benchmarking são:

- Funcionamento das operações

- Rendimento (custos, produtividade, etc.)

- Gestão da segurança. Higiene e meio ambiente

- Flexibilidade e agilidade da atuação mercadológica

- Etc.

\subsection{BENCHMARKING AMBIENTAL:}

A conjugação de melhor desempenho ambiental com uma maior competitividade no mercado só é possível com mudanças tecnológicas e gerenciais. Daí a importância da adoção do Benchmarking Ambiental para a melhoria contínua das boas práticas ambientais encontradas nos Sistemas de Gestão Ambiental das Empresas.

\section{GESTÃO AMBIENTAL:}

A Gestão Ambiental, é definida como um conjunto de princípios, estratégias e diretrizes de ações e procedimentos para preservar a integridade dos meios físico e biótico, bem como a dos grupos sociais que deles dependem. A Gestão Ambiental visa ordenar as atividades humanas para que estas originem o menor impacto possível sobre o meio. Esta ordem vai desde a escolha das melhores técnicas até o cumprimento da legislação e a alocação correta de recursos humanos e financeiros.

Já o Benchmarking ambiental é praticado como uma forma de aprendizado por meio de comparações competitivas com ênfase nos processos e resultados das empresas e organizações que são reconhecidas como representantes das melhores práticas ambientais. Simplificando, Benchmarking ambiental é o processo de comparação entre as práticas que aplicam empresas da mesma ou distinta atividade, com o objetivo do aprimoramento e melhoria contínua da gestão ambiental empresarial.

\subsection{VANTAGENS DA GESTÃO AMBIENTAL :}


Uma empresa que adota as boas práticas ambientais contidas nos Sistemas de Gestão Ambiental empresarial, tem em seu quadro, profissionais esclarecidos e envolvidos com a questão ambiental. Um Profisssional consciente ambientalmente, compreende que poluição é na maioria das vezes, baixa eficiência do processo produtivo, gerado pelas perdas de matérias primas, de energia e outros insumos. Prejuízo em dose tripla:

- Agride nossa qualidade de vida

- Gera custos advindos das perdas e penalidades

- Coloca a em risco a imagem da empresa perante o mercado e outros públicos de interesse

Hoje, encontramos posturas e resultados diferentes das organizações para responderem aos desafios ambientais. Podemos dizer que basicamente existem 4 formas de atuação que adotam:

1. Resolver os problemas quando eles ocorrem

2. Cumprir com as exigências legais e regulatórias

3. Praticar uma gestão ambiental consistente, onde ações são tomadas apenas depois de considerados seus efeitos ao meio ambiente

4. Perseguir o desenvolvimento sustentável, isto é, adotar ações que tragam efeitos benéficos a longo-prazo na economia, ao meio ambiente e a sociedade.

Evidentemente que as empresas que adotam as boas práticas ambientais são mais bem sucedidas nas metas de prevenção e de melhoria contínua para identificar formas de redução de custos, e oportunidades para atender um mercado crescente para os produtos e serviços tecnologicamente mais limpos.

\subsection{Principais práticas ambientais empresariais:}

- RESPONSIBLE CARE ou Atuação Responsável - Uma iniciativa das industrias químicas para controle e prevenção da poluição e dos impactos 
ambientais - Canadian Chemical Producers Association - CCPA- Canadá 1985 - (Brasil: ABIQUIM)

- ISO SÉRIE 14000: Conjunto de normas internacionais que tem por objetivo prover as organizações os elementos de um sistema de gestão ambiental, possível de integração com outros requisitos de gestão, de forma a auxiliá-las a alcançar seus objetivos ambientais e econômicos. A ISO 14001contém normas e regulamentos para prevenir e controlar os processos de produção de forma a não impactar o meio ambiente -International Standardization Organization - Genebra/Suiça (Brasil: ABNT)

- SA 8000: A SA 8000 regulamenta questões referentes ao trabalho infantil, ao trabalho forçado, à saúde e à segurança, à liberdade de sindicalização e o direito de negociação coletiva, à discriminação, às práticas disciplinares, às horas de trabalho, à remuneração, ao sistema de gestão de responsabilidade social, etc. Sua certificação constitui a materialização de um consenso éticonormativo sobre a responsabilidade social das empresas, sob as prerrogativas da Declaração dos Direitos Humanos das Nações Unidas. A responsabilidade dessa iniciativa partiu do "CEPA - Council on Economic Priorities Agency (Conselho da Agência de Prioridades Econômicas dos EUA)", e representa um novo padrão de certificação que, embora recente, já é conhecido mundialmente.

- GESTÃO INTEGRADA: É a combinação de processos, procedimentos e práticas adotadas por uma organização para implementar suas políticas e atingir seus objetivos de forma mais eficiente do que através de múltiplos sistemas de gestão. Na integração de elementos de sistemas de gestão, considerando-se as dimensões qualidade, meio ambiente, saúde e segurança no trabalho, temos a congregação das normas ISO 9001, ISO 14001, e OSHAS 18001.

- LICENCIAMENTO AMBIENTAL: É um dos mais eficazes instrumentos da política ambiental para a viabilização do desenvolvimento sustentado. É um ato administrativo pelo qual o órgão ambiental competente estabelece as condições, restrições e medidas de controle ambiental que deverão ser 
obedecidas para a liberação da: LP(Licença Prévia), LI (Licença de Instalação) e LO (Licença de Operação) .

- EIA/RIMA: Estudos de Impacto Ambiental e Relatório de Impacto ambiental: Regulamentado através da Resolução CONAMA 001/86, que estabelece a obrigatoriedade da elaboração e apresentação de EIA/RIMA para licenciamento de empreendimentos que possam modificar o meio ambiente.

- EDUCAÇÃO AMBIENTAL: Processo por meio dos quais o indivíduo e a coletividade constroem valores sociais, conhecimentos, habilidades, atitudes e competências voltadas para a conservação do meio ambiente, bem de uso comum do povo, essencial à sadia qualidade de vida e sua sustentabilidade. (art.1 ${ }^{\circ}$, Lei Federal n ${ }^{\circ}$ 9.795, de 27/4/99)

- DESENVOLVIMENTO SUSTENTÁVEL: O tratamento político que integra desenvolvimento e sustentabilidade, é um processo que supõe a conciliação entre diferentes opções e valores vigentes na sociedade. A convergência entre os propósitos das áreas econômica e ecológica, que privilegiam a conservação e o longo prazo dos sistemas econômico e natural, é base do desenvolvimento sustentável.

- MARKETING VERDE: Processo através do qual a economia sustentável é integrada à sociedade, atraindo clientes de forma a atender às suas necessidades bem como aos objetivos da organização, tornando perene sua existência.

\section{PESQUISA GESTÃO SÓCIO-AMBIENTAL NA EMPRESA:}

Em Janeiro de 2003, foi realizada uma pesquisa com 286 empresas sobre gestão sócio-ambiental na empresa com o objetivo de conhecer o grau de amadurecimento e o nível interesse pelo tema Benchmarking Ambiental. Nesta pesquisa, 89\% das empresas manifestaram interesse na prática do Benchmarking Ambiental apontando inclusive os principais temas de interesse. (Fonte: http://www.maisprojetos.com.br/agenda/pesquisa.htm 


\subsection{PESQUISA GESTÃO SÓCIO-AMBIENTAL NA EMPRESA -}

RESULTADO

SUA EMPRESA TEM SGA - SISTEMA DE GESTÃO AMBIENTAL?

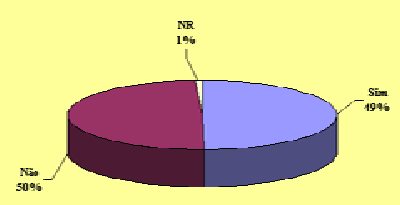

Fonte: Mais Projetos, mar/2003.

HÁ QUANTO TEMPO A EMPRESA POSSUI SGA?

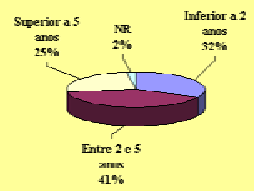

Fonte: Mais Projetos, mar/2003.

QUANTOS COLABORADORES TÊM A EQUIPE SGA?

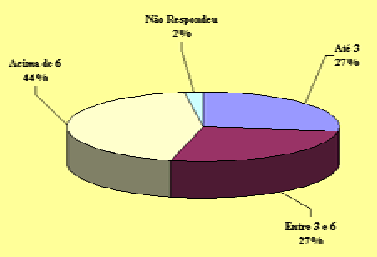

Fonte: Mais Projetos, mar/2003.

A EMPRESA POSSUI CERTIFICAÇÃO ISO 14001?

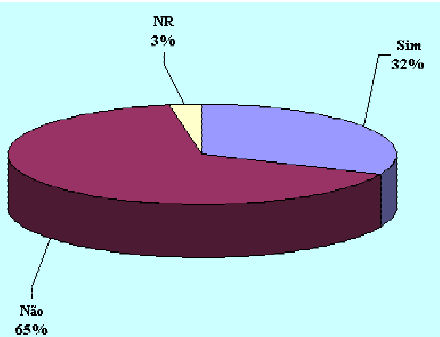

Fonte: Mais Projetos, mar/2003. 


\section{HÁ QUANTO TEMPO POSSUI CERTIFICAÇÃO?}

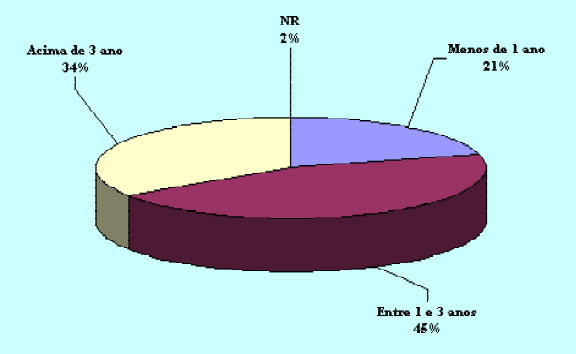

Fonte: Mais Projetos, mar/2003.

\section{TEM INTERESSE EM BENCHMARKING AMBIENTAL?}

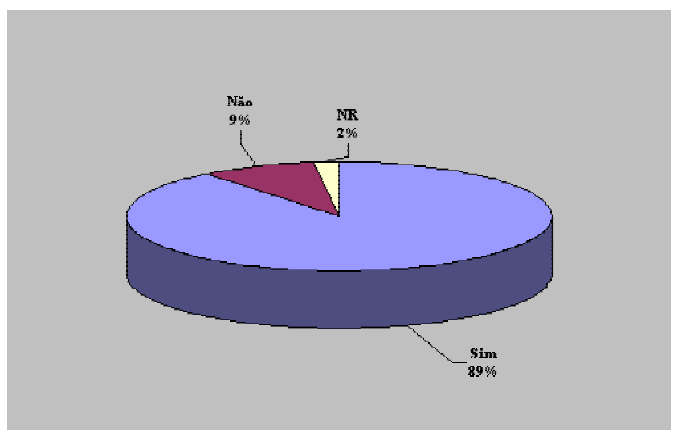

Fonte: Mais Projetos, mar/2003.

QUAIS SÃO AS PRINCIPAIS ÁREAS E TEMAS DE INTERESSE PARA BENCHMARKING

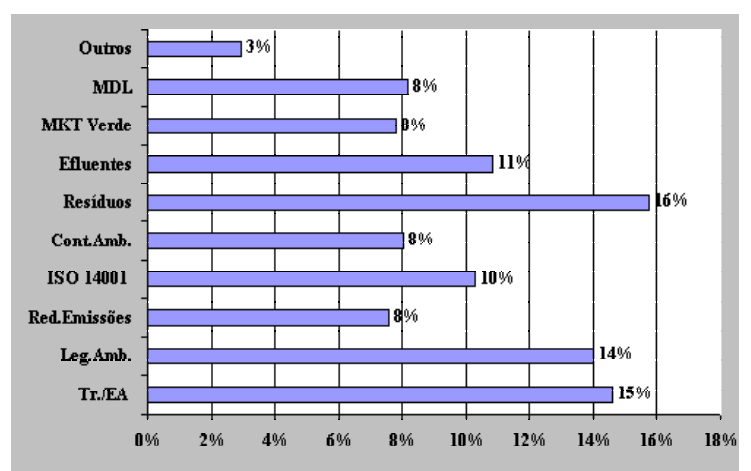

Fonte: Mais Projetos, mar/2003.

PERFIL DE PÚBLICO 


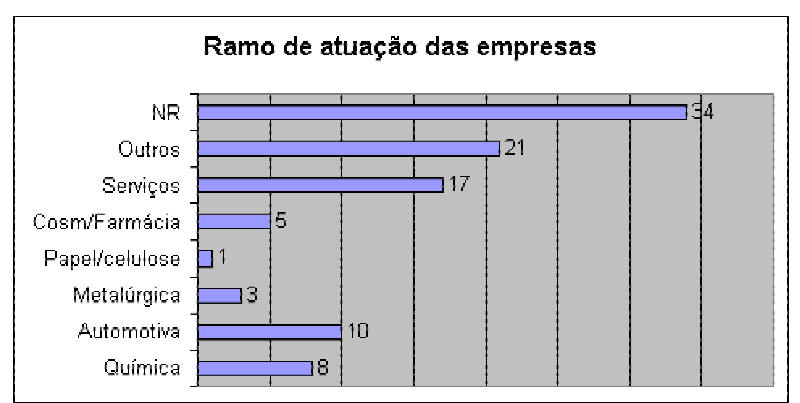

Fonte: Mais Projetos, mar/2003.

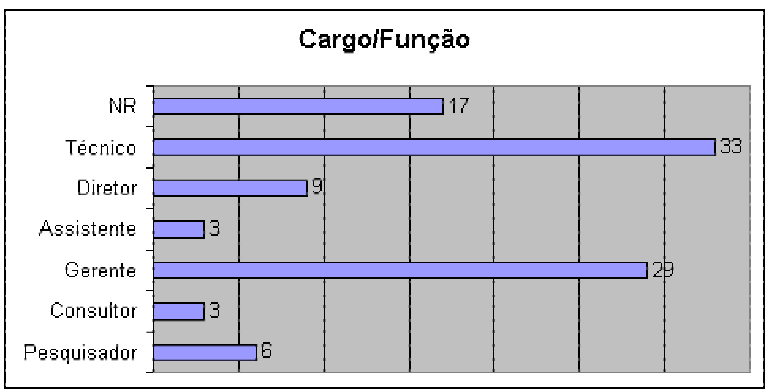

Fonte: Mais Projetos, março/2003.

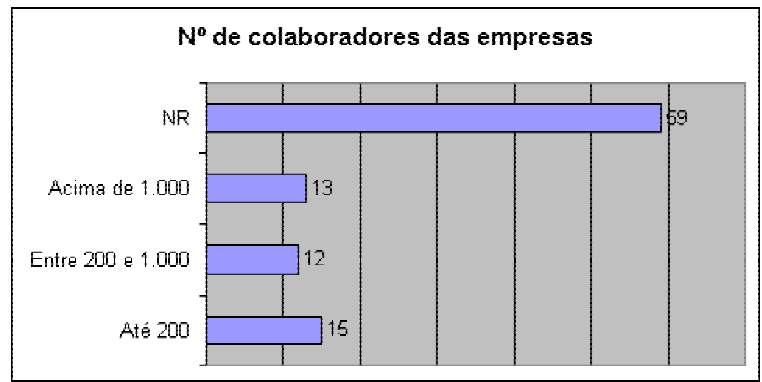

Fonte: Mais Projetos, março/2003.

PERFORMANCE

\begin{tabular}{|c|c|c|l|}
\hline Data & $\begin{array}{c}\mathbf{n}^{0} \text { e-mails } \\
\text { distribuídos }\end{array}$ & $\begin{array}{c}\mathbf{n}^{0} \text { de e-mails } \\
\text { recebidos }\end{array}$ & Fator \\
\hline $\mathbf{2 8} / \mathbf{2} / \mathbf{0 3}$ & $\mathbf{1 1 . 2 0 0}$ & $\mathbf{2 8 6}$ & $\mathbf{0 , 0 2 5 5 3 6}$ \\
\hline
\end{tabular}

\subsection{BENCHMARKING AMBIENTAL BRASILEIRO}

Em virtude do resultado desta pesquisa foi organizado o $1^{\circ}$ Benchmarking Ambiental Brasileiro (Ver: http://www.maisprojetos.com.br/bench/ ), com o objetivo de reunir, selecionar e premiar as melhores práticas ambientais, e assim apresentar uma amostragem de como as empresas e instituições brasileiras estão atuando e que 
medidas estão tomando para contribuir com a qualidade e a segurança ambiental no país. Como resultado, mais de 30 inscrições de "cases"de empresas e instituições localizadas em vários pontos do país que foram avaliados por uma Comissão Técnica especialmente composta para esta finalidade, e que foram selecionados em 02 categorias: Premiados e Menção Honrosa.

A Comissão Técnica privilegiou cases de empresas e instituições que praticam gestão ambiental além da exigência legal e que compartilham o resultado não só com seu público interno, mas com toda a sociedade.

Os cases selecionados portanto, foram aqueles que excederam e contribuíram com o desenvolvimento sustentável e o uso racional dos recursos naturais, adotando práticas que promoveram a melhoria da qualidade do ar, a redução de emissões, a racionalização e conservação dos Recursos Hídricos, a consciência ambiental, entre outros.

Como resultado, 14 "cases" com enfoque em diversos itens da gestão ambiental foram validados como referências em suas áreas de atuação. Veja tabela abaixo: (Fonte: http://www.maisprojetos.com.br/bench/vencedores.htm )

\begin{tabular}{|c|c|c|}
\hline \multicolumn{3}{|c|}{$\begin{array}{c}\text { VENCEDORES DO } 1^{\circ} \text { BENCHMARKING AMBIENTAL } \\
\text { BRASILEIRO - Agosto } 2003 \\
\end{array}$} \\
\hline \multicolumn{3}{|c|}{ CATEGORIA CASES PREMIADOS } \\
\hline Colocação & Empresa/Instituição & Case \\
\hline $1^{\circ}$ & $\begin{array}{l}\text { GLOBAL TELECOM } \\
\text { (VIVO) - PR }\end{array}$ & Gerenciamento Ambiental Avançado \\
\hline $2^{\circ}$ & EMBRATEL - RJ & Agenda 21 Embratel \\
\hline $3^{\circ}$ & \begin{tabular}{|l|} 
PREFEITURA DE POTIM \\
- SP
\end{tabular} & Potim Mais Verde \\
\hline $4^{\circ}$ & $\begin{array}{l}\text { SONOCO DO BRASIL - } \\
\text { PR }\end{array}$ & $\begin{array}{l}\text { Recuperação da Mata Ciliar do Ribeirão } \\
\text { Quati }\end{array}$ \\
\hline $5^{\circ}$ & VM FLORESTAL - MG & $\begin{array}{l}\text { Viabilização e Sustentabilidade de uma fonte } \\
\text { de energia renovável para a Indústria }\end{array}$ \\
\hline $6^{\circ}$ & CARAIBA METAIS - BA & Fenômeno das Andorinhas \\
\hline $7^{\circ}$ & AMBEV - SP & $\begin{array}{l}\text { Gestão de Recursos Hídricos e Reciclagem } \\
\text { de Resíduos }\end{array}$ \\
\hline $8^{\circ}$ & UNIMED Brasil - SP & $\begin{array}{l}\text { Manual de Responsabilidade Social } \\
\text { Eticamente Correto }\end{array}$ \\
\hline
\end{tabular}




\begin{tabular}{||c||c||c||}
\hline \multicolumn{3}{|c||}{ CATEGORIA CASES MENÇÃO HONROSA } \\
\hline \hline Colocação & Empresa/Instituição & \multicolumn{1}{c||}{ Case } \\
\hline \hline $1^{\circ}$ & $\begin{array}{l}\text { COOPERATIVA } \\
\text { AURORA - SC }\end{array}$ & A Turminha da Reciclagem \\
\hline \hline $2^{\circ}$ & SABESP - SP & $\begin{array}{l}\text { Gestão Ambiental por Parcerias (Caso } \\
\text { Morada dos Pássaros) }\end{array}$ \\
\hline \hline $3^{\circ}$ & CEFETSC - SC & $\begin{array}{l}\text { Um enfoque empreendedor em conservação } \\
\text { e eficiência energética no CEFETSC }\end{array}$ \\
\hline \hline $4^{\circ}$ & $\begin{array}{l}\text { SOCIEDADE } \\
\text { ALPHAVILLE 11 - SP }\end{array}$ & Terra Limpa \\
\hline \hline $5^{\circ}$ & $\begin{array}{l}\text { CASA DA } \\
\text { COMUNICAÇÃO - BA }\end{array}$ & Manguezal do Rio Passa Vaca \\
\hline \hline $6^{\circ}$ & $\begin{array}{l}\text { FLUÍDOS DA AMAZONIA } \\
\text { PA }\end{array}$ & Ecoparcerias - Facilitando o Trabalho \\
\hline \hline
\end{tabular}

\section{CONCLUSÕES:}

Enquanto que a média dos resultados dos programas de melhoria contínua está em torno de 7\%, com a inclusão da prática do Benchmarking chega-se até $300 \%$ de resultado nos programas de melhoria contínua, segundo o Prof. D. Enrique de la Rica, Director de la Escuela Europea de Estudios Universitarios y de Negocios (ESEUNE www.eseune.edu Neguri, Getxo - Bizkaia - España),

Também é necessário identificar os melhores em suas áreas de atuação para praticar o Benchmarking, que neste caso, a realização do $1^{\mathrm{o}}$ Benchmarking Ambiental Brasileiro cumpriu este papél de identificação e validação dos melhores cases de "gestão ambiental".

O $1^{\text {o }}$ Benchmarking Ambiental Brasileiro forneceu subsídios para uma amostragem da gestão ambiental praticada nas empresas brasileiras, já que selecionou e premiou "cases" de empresas e instituições de 07 estados brasileiros, dos 03 setores da economia, de vários portes e ramos de atividades englobando tanto segmento produtivo quanto o de serviços. Outro ponto a ser destacado é resultado que vem comprovar que gestão ambiental consistente independe do porte e ramo de atividade, e sim, de conhecimento, atitude e prática, mesmo porque entre os premiados encontram-se empresas e instituições de vários portes e ramos de atividades. 
Desta forma, evidencia-se o resultado positivo da pratica do Benchmarking Ambiental, que não só contribui com o avanço técnico-gerencial da gestão ambiental corporativa como também acelera o processo de amadurecimento das empresas, sociedade e profissionais nesta questão, conferindo assim, competitividade as empresas e instituições praticantes.

\section{REFERÊNCIAS BIBLIOGRAFICAS:}

CASCINO, Fábio. Jacobi, Pedro. Oliveira, José Flávio de. EDUCAÇÃO, MEIO AMBIENTE E CIDADANIA: Reflexões e experiências. São Paulo, Governo do Estado de São Paulo, 1998.p.11 - 32

DIAS, Genebaldo Freire. Educação Ambiental: Princípios e Práticas. Brasília, Editora Gaia, 1992.

CÂMARA DE COMÉRCIO E INDUSTRIA BRASIL-ALEMANHA, $1^{\circ}$ Guia de Tecnologias Ambientais Brasil-Alemanha. São Paulo, 1998. p.114-116.

JURAN INSTITUTE. BENCHMARKING. Disponível em http://www.juran.es/benchmarking.html . Acesso em 10/12/2003

GOETSCH/David L./DAVIS, Stanley, Introduction to Total Quality, Editorial Merrill, capitulo 13. Disponível em http://mailweb.udlap.mx/ jtambore/benchmarking/benchmarking.html. Acesso em $09 / 12 / 2003$

GANAR OPCIONES. LA RICA, Enrique de, Benchmarking como herramienta competitiva. Disponível em http://ganaropciones.com.ar/benchmarking.htm

ENCICLOPEDIA-MEX. PARRA, Sergio Bojalil, BENCHMARKING - LA BÚSQUEDA DE LAS MEJORES PRÁCTICAS. Disponível em http://enciclopediamex.galeon.com/CAdPracticas.htm Acesso em 09/12/2003.

MAIS PROJETOS. Pesquisa Gestão Sócio-ambiental na empresa. Disponível em http://www.maisprojetos.com.br/index.htm Acesso em 09/12/2003 